TRANSACTIONS OF THE

AMERICAN MATHEMATICAL SOCIETY

Volume 362, Number 6, June 2010, Pages 2901-2922

S 0002-9947(10)04695-7

Article electronically published on January 5, 2010

\title{
LAYER POTENTIAL TECHNIQUES IN SPECTRAL ANALYSIS. PART I: COMPLETE ASYMPTOTIC EXPANSIONS FOR EIGENVALUES OF THE LAPLACIAN IN DOMAINS WITH SMALL INCLUSIONS
}

\author{
HABIB AMMARI, HYEONBAE KANG, MIKYOUNG LIM, AND HABIB ZRIBI
}

\begin{abstract}
We provide a rigorous derivation of new complete asymptotic expansions for eigenvalues of the Laplacian in domains with small inclusions. The inclusions, somewhat apart from or nearly touching the boundary, are of arbitrary shape and arbitrary conductivity contrast vis-à-vis the background domain, with the limiting perfectly conducting inclusion. By integral equations, we reduce this problem to the study of the characteristic values of integral operators in the complex plane. Powerful techniques from the theory of meromorphic operator-valued functions and careful asymptotic analysis of integral kernels are combined for deriving complete asymptotic expansions for eigenvalues. Our asymptotic formulae in this paper may be expected to lead to efficient algorithms not only for solving shape optimization problems for Laplacian eigenvalues but also for determining specific internal features of an object based on scattering data measurements.
\end{abstract}

\section{INTRODUCTION}

Let $\Omega$ be a bounded domain in $\mathbb{R}^{d}, d \geq 2$, with a connected Lipschitz boundary $\partial \Omega$. Let $\nu$ denote the unit outward normal to $\partial \Omega$. Suppose that $\Omega$ contains a small inclusion $D$, of the form $D=z+\epsilon B$, where $B$ is a bounded Lipschitz domain in $\mathbb{R}^{d}$ containing the origin. We also assume that the "background" is homogeneous with conductivity 1 . The inclusions, somewhat apart from or nearly touching the boundary, are of arbitrary conductivity contrast vis-à-vis the background domain, with the limiting perfectly conducting case.

Our goal is to find complete asymptotic expansions for the eigenvalues of such a domain which had not been established before this work, with the intention of using the expansions as an aid in identifying the inclusions. That is, we would like to find a method for determining the locations and/or shape of small inclusions by taking eigenvalue measurements. In its most general form the inverse scattering problem is severely ill-posed and nonlinear. This is the main obstacle to finding noniterative reconstruction algorithms with limited scattering data. If, however, in advance we have additional structural information about the conductivity profile, then we may be able to determine from scattering data specific features about the conductivity distribution with a satisfactory resolution. One such type of knowledge

Received by the editors January 27, 2006 and, in revised form, December 9, 2007.

2000 Mathematics Subject Classification. Primary 35B30.

Key words and phrases. Eigenvalue problem, Laplacian, small inclusion, full-asymptotic expansions, boundary integral equation. 
could be that the body consists of a smooth background containing a number of unknown small inclusions with a significantly different conductivity. Our method of asymptotic expansions of small volume inclusions may provide a useful framework to accurately and efficiently reconstruct the location and geometric features of the inclusions in a stable way, even for moderately noisy scattering data. Our formulae in this paper may also be expected to lead to efficient algorithms for solving shape optimization problems for Laplacian eigenvalues. See [24].

Rauch and Taylor [31] have shown that the spectrum of a bounded domain does not change after imposing Dirichlet conditions on a compact subset of capacity zero. After that, many people have studied the asymptotic expansion of the eigenvalues for the case of small holes with the Dirichlet or the Neumann boundary condition. In particular, Ozawa provided in a series of papers [25]-30] leading-order terms in eigenvalue expansions; see also [37] and [21. Besson [15] has proved the existence of a complete expansion of the eigenvalue perturbation in the two-dimensional case. Courtois [16] has established a perturbation theory for the Dirichlet spectrum in a compactly perturbed domain in terms of the capacity of the compact perturbation. We shall also mention, in connection with eigenvalue changes under variation of domains, the works by Kato 20, Sanchez Hubert and Sanchez Palencia 33, Ward and Keller 37, Gadyl'shin and Il'in [18, Daners [17, McGillivray 22, and Noll 23.

In this paper we provide a rigorous derivation of new complete asymptotic expansions for eigenvalues of the Laplacian in domains with small inclusions. The inclusions are of arbitrary shape and of arbitrary conductivity contrast. A key difference in our work is the approach we develop: a general and unified boundary integral approach with rigorous justification based on the generalized Rouché theorem. By using layer potential techniques we show that the square roots of the eigenvalues are the characteristic values of meromorphic operator-valued functions that are of Fredholm type with index 0. We then proceed from the generalized Rouché theorem to construct their complete asymptotic expressions. Our idea is to reduce the eigenvalue problem to the study of characteristic values of systems of certain integral operators. Here, we confine our attention to the eigenvalues of the Neumann boundary value problem in the bounded domain $\Omega$. The eigenvalue problem with the Dirichlet boundary condition is of equal interest. The asymptotic results for the eigenvalues in such cases can be obtained with only minor modifications of the techniques presented here, while the rigorous derivations of similar asymptotic formulae for the full Maxwell equations or for the equations of linear elasticity require further work.

\section{Notation AND PRELIMINARIES}

2.1. The generalized Rouché theorem. In this work the approach we develop is a boundary integral technique with rigorous justification based on the generalized Rouché theorem. For the reader's convenience we recall this theorem due to Gohberg and Sigal in [19. We begin by collecting some notation.

Let $\mathcal{H}$ and $\mathcal{H}^{\prime}$ be two Banach spaces, and let $\mathcal{L}\left(\mathcal{H}, \mathcal{H}^{\prime}\right)$ be the algebra of all bounded vector-valued functions acting from $\mathcal{H}$ into $\mathcal{H}^{\prime}$.

Let $\omega_{0}$ be a fixed complex value in $\mathbb{C}$. We denote by $\mathcal{A}(\omega)$ an operator-valued function acting from $V_{\delta}\left(\omega_{0}\right)$ into $\mathcal{L}\left(\mathcal{H}, \mathcal{H}^{\prime}\right)$, where $V_{\delta}\left(\omega_{0}\right)$ is a disc of center $\omega_{0}$ and radius $\delta>0$. 
$\omega_{0}$ is called a characteristic value of $\mathcal{A}(\omega)$ if

(i) $\mathcal{A}(\omega)$ is holomorphic in some neighborhood of $\omega_{0}$, except possibly at this point itself;

(ii) there exists a vector-valued function $\phi(\omega): V_{\delta}\left(\omega_{0}\right) \rightarrow \mathcal{H}$ holomorphic at $\omega_{0}$ and that verifies $\phi\left(\omega_{0}\right) \neq 0$, such that $\mathcal{A}(\omega) \phi(\omega)$ is holomorphic at $\omega_{0}$ and vanishes at this point. $\phi(\omega)$ is called a root function of $\mathcal{A}(\omega)$ associated with $\omega_{0}$, and the vector $\phi_{0}=\phi\left(\omega_{0}\right)$ is called an eigenvector. The closure of the linear set of eigenvectors corresponding to $\omega_{0}$ is denoted by $\operatorname{Ker} \mathcal{A}\left(\omega_{0}\right)$.

Suppose that $\omega_{0}$ is a characteristic value of the function $\mathcal{A}(\omega)$ and $\phi(\omega)$ is a root function satisfying (ii). Then there exists a number $m(\phi) \geq 1$ and a vector-valued function $\psi(\omega): V_{\delta}\left(\omega_{0}\right) \rightarrow \mathcal{H}$ holomorphic such that

$$
\mathcal{A}(\omega) \phi(\omega)=\left(\omega-\omega_{0}\right)^{m(\phi)} \psi(\omega), \quad \psi\left(\omega_{0}\right) \neq 0 .
$$

The number $m(\phi)$ is called the multiplicity of the root function $\phi(\omega)$. Let $\phi_{0}$ be an eigenvector corresponding to $\omega_{0}$ and let

$$
\mathcal{R}\left(\phi_{0}\right)=\left\{m(\phi) ; \phi(\omega) \text { is a root function such that } \phi\left(\omega_{0}\right)=\phi_{0}\right\} .
$$

Then by rank of $\phi_{0}$ we mean $\operatorname{rank}\left(\phi_{0}\right)=\max \mathcal{R}\left(\phi_{0}\right)$.

Suppose that $n=\operatorname{dimKer} \mathcal{A}\left(\omega_{0}\right)<+\infty$ and that the ranks of all vectors in $\operatorname{Ker} \mathcal{A}\left(\omega_{0}\right)$ are finite. A system of eigenvectors $\phi_{0}^{j}, j=1, \ldots, n$, is called a canonical system of eigenvectors of $\mathcal{A}(\omega)$ associated to $\omega_{0}$ if the ranks possess the following property: $\operatorname{rank}\left(\phi_{0}^{j}\right)$ is the maximum of the ranks of all eigenvectors in some direct complement in $\operatorname{dimKer} \mathcal{A}\left(\omega_{0}\right)$ of the linear span of the vectors $\phi_{0}^{1}, \ldots, \phi_{0}^{j-1}$. Let $r_{j}=\operatorname{rank}\left(\phi_{0}^{j}\right)$. We call

$$
N\left(\mathcal{A}\left(\omega_{0}\right)\right)=\sum_{j=1}^{n} r_{j}
$$

the null multiplicity of the characteristic value $\omega_{0}$ of $\mathcal{A}(\omega)$. If $\omega_{0}$ is not a characteristic value of $\mathcal{A}(\omega)$, we put $N\left(\mathcal{A}\left(\omega_{0}\right)\right)=0$.

Suppose that $\mathcal{A}^{-1}(\omega)$ exists and is holomorphic in some neighborhood of $\omega_{0}$, except possibly at this point itself. Then the number

$$
M\left(\mathcal{A}\left(\omega_{0}\right)\right)=N\left(\mathcal{A}\left(\omega_{0}\right)\right)-N\left(\mathcal{A}^{-1}\left(\omega_{0}\right)\right)
$$

is called the multiplicity of the characteristic value $\omega_{0}$ of $\mathcal{A}(\omega)$. Suppose that $\omega_{1}$ is a pole of the operator-valued function. The Laurent expansion of $\mathcal{A}(\omega)$ in $\omega_{1}$ is given by

$$
\mathcal{A}(\omega)=\sum_{j \geq-s}\left(\omega-\omega_{1}\right)^{j} A_{j} .
$$

If in the last expression the operators $A_{-j}, j=1, \ldots, s$, are finite-dimensional, then $\mathcal{A}(\omega)$ is called finitely meromorphic at $\omega_{1}$.

The operator-valued function $\mathcal{A}(\omega)$ is said to be of Fredholm type at the point $\omega_{1}$ if the operator $A_{0}$ in the last expansion is a Fredholm operator. If $\mathcal{A}(\omega)$ is holomorphic at the point $\omega_{0}$ and the operator $\mathcal{A}\left(\omega_{0}\right)$ is invertible, then $\omega_{0}$ is called a regular point of $\mathcal{A}(\omega)$.

The point $\omega_{0}$ is called a normal point of $\mathcal{A}(\omega)$ if there exists a constant $0<\delta_{0}$ such that $\mathcal{A}(\omega)$ is finitely meromorphic and of Fredholm type at $\omega_{0}$ and all the points of a disc of center $\omega_{0}$ and radius $\delta_{0}>0$ except $\omega_{0}$ are regular for $\mathcal{A}(\omega)$. 
Lemma 2.1. Every normal point $\omega_{0}$ of $\mathcal{A}(\omega)$ is a normal point of $\mathcal{A}^{-1}(\omega)$. If, in addition, $\omega_{0}$ is a pole of either $\mathcal{A}(\omega)$ or $\mathcal{A}^{-1}(\omega)$, then it is a characteristic value of finite multiplicity of the other.

Let $\partial V$ be the contour bounding the domain $V$. An operator-valued function $\mathcal{A}(\omega)$ which is finitely meromorphic and of Fredholm type in $V$ and continuous at $\partial V$ is called normal with respect to $\partial V$ if the operator $\mathcal{A}(\omega)$ is invertible in $\bar{V}$, except for a finite number of points of $V$ which are normal points of $\mathcal{A}(\omega)$. Now, if $\mathcal{A}(\omega)$ is normal with respect to the contour $\partial V$ and $\omega_{i}, i=1, \ldots, \sigma$, are all its characteristic values and poles lying in $V$, we put

$$
\mathcal{M}(\mathcal{A}(\omega) ; \partial V)=\sum_{i=1}^{\sigma} M\left(\mathcal{A}\left(\omega_{i}\right)\right) .
$$

Theorem 2.2. Suppose that the operator-valued $\mathcal{A}(\omega)$ is normal with respect to $\partial V$; then we have

$$
\mathcal{M}(\mathcal{A}(\omega) ; \partial V)=\frac{1}{2 i \pi} \operatorname{tr} \int_{\partial V} \mathcal{A}^{-1}(\omega) \frac{d}{d \omega} \mathcal{A}(\omega) d \omega
$$

By 'tr' we mean the trace of the operator which is the sum of all its nonzero characteristic values; see [19, p. 609] for an exact statement. We mention the following property of the trace:

$$
\operatorname{tr} \int_{\partial V} \mathcal{A}(\omega) \mathcal{B}(\omega) d \omega=\operatorname{tr} \int_{\partial V} \mathcal{B}(\omega) \mathcal{A}(\omega) d \omega
$$

where $\mathcal{A}(\omega)$ and $\mathcal{B}(\omega)$ are operator-valued functions which are finitely meromorphic in the neighborhood $V$ of $\omega_{0}$, which contains no poles of $\mathcal{A}(\omega)$ and $\mathcal{B}(\omega)$ other than $\omega_{0}$.

The operator generalization of the Rouché theorem is stated below.

Theorem 2.3. Let $\mathcal{A}(\omega)$ be an operator-valued function which is normal with respect to $\partial V$. If an operator-valued function $S(\omega)$ which is finitely meromorphic in $V$ and continuous at $\partial V$ satisfies the condition

$$
\left|\mathcal{A}^{-1}(\omega) S(\omega)\right|_{\mathcal{L}(\mathcal{H}, \mathcal{H})}<1, \quad \omega \in \partial V,
$$

then $\mathcal{A}(\omega)+S(\omega)$ is also normal with respect to $\partial V$, and

$$
\mathcal{M}(\mathcal{A}(\omega) ; \partial V)=\mathcal{M}(\mathcal{A}(\omega)+S(\omega) ; \partial V) .
$$

The generalization of the Steinberg theorem is given by the following.

Theorem 2.4. Suppose that $\mathcal{A}(\omega)$ is an operator-valued function which is finitely meromorphic and of Fredholm type in the domain $V$. If the operator $\mathcal{A}(\omega)$ is invertible at one point of $V$, then $\mathcal{A}(\omega)$ has a bounded inverse for all $\omega \in V$, except possibly for certain isolated points.

Finally, the following result due to Gohberg and Sigal in [19] is central.

Theorem 2.5. Suppose that $\mathcal{A}(\omega)$ is an operator-valued function which is normal with respect to $\partial V$. Let $f(\omega)$ be a scalar function which is analytic in $V$ and continuous in $\bar{V}$. Then

$$
\frac{1}{2 i \pi} \operatorname{tr} \int_{\partial V} f(\omega) \mathcal{A}^{-1}(\omega) \frac{d}{d \omega} \mathcal{A}(\omega) d \omega=\sum_{j=1}^{\sigma} M\left(\mathcal{A}\left(\omega_{j}\right)\right) f\left(\omega_{j}\right),
$$


where $\omega_{j}, j=1, \ldots, \sigma$, are all the points in $V$ which are either poles or characteristic values of $\mathcal{A}(\omega)$.

2.2. Layer potentials for the Helmholtz equation. We will develop a boundary integral formulation for solving the eigenvalue problem. The integral equations applying to this problem will be obtained from a study of the layer potentials for the Helmholtz equation.

For $\omega>0$, a fundamental solution $\Gamma_{\omega}(x)$ to the Helmholtz operator $\Delta+\omega^{2}$ in $\mathbb{R}^{d}, d=2,3$, is given by

$$
\Gamma_{\omega}(x):= \begin{cases}-\frac{i}{4} H_{0}^{(1)}(\omega|x|), & d=2, \\ -\frac{e^{i \omega|x|}}{4 \pi|x|}, & d=3,\end{cases}
$$

for $x \neq 0$, where $H_{0}^{(1)}$ is the Hankel function of the first kind of order 0 .

For a bounded Lipschitz domain $D$ in $\mathbb{R}^{d}$ and $\omega>0$, let $\mathcal{S}_{D}^{\omega}$ and $\mathcal{D}_{D}^{\omega}$ be the single and double layer potentials defined by $\Gamma_{\omega}$, that is,

$$
\begin{aligned}
& \mathcal{S}_{D}^{\omega} \varphi(x):=\int_{\partial D} \Gamma_{\omega}(x-y) \varphi(y) d \sigma(y), \quad x \in \mathbb{R}^{d}, \\
& \mathcal{D}_{D}^{\omega} \varphi(x):=\int_{\partial D} \frac{\partial \Gamma_{\omega}(x-y)}{\partial \nu_{y}} \varphi(y) d \sigma(y), \quad x \in \mathbb{R}^{d} \backslash \partial D,
\end{aligned}
$$

for $\varphi \in L^{2}(\partial D)$.

The following formulae give the jump relations obeyed by the double layer potential and by the normal derivative of the single layer potential on general Lipschitz domains:

$$
\begin{gathered}
\left.\frac{\partial\left(\mathcal{S}_{D}^{\omega} \varphi\right)}{\partial \nu}\right|_{ \pm}(x)=\left( \pm \frac{1}{2} I+\left(\mathcal{K}_{D}^{\omega}\right)^{*}\right) \varphi(x) \quad \text { a.e. } x \in \partial D, \\
\left.\left(\mathcal{D}_{D}^{\omega} \varphi\right)\right|_{ \pm}(x)=\left(\mp \frac{1}{2} I+\mathcal{K}_{D}^{\omega}\right) \varphi(x) \quad \text { a.e. } x \in \partial D,
\end{gathered}
$$

for $\varphi \in L^{2}(\partial D)$, where $\mathcal{K}_{D}^{\omega}$ is the operator defined by

$$
\mathcal{K}_{D}^{\omega} \varphi(x):=\text { p.v. } \int_{\partial D} \frac{\partial \Gamma_{\omega}(x-y)}{\partial \nu_{y}} \varphi(y) d \sigma(y),
$$

and $\left(\mathcal{K}_{D}^{\omega}\right)^{*}$ is the $L^{2}$-adjoint of $\mathcal{K}_{D}^{\omega}$, that is,

$$
\left(\mathcal{K}_{D}^{\omega}\right)^{*} \varphi(x):=\text { p.v. } \int_{\partial D} \frac{\partial \Gamma_{\omega}(x-y)}{\partial \nu_{x}} \varphi(y) d \sigma(y) .
$$

Here p.v. stands for the Cauchy principal value. The singular integral operators $\mathcal{K}_{D}^{\omega}$ and $\left(\mathcal{K}_{D}^{\omega}\right)^{*}$ are known to be bounded on $L^{2}(\partial D)$.

Let $0<\mu_{1} \leq \mu_{2} \leq \ldots$ be the eigenvalues of $-\Delta$ in $\Omega$ with the Neumann condition on $\partial \Omega$. Let $u_{j}$ denote the normalized eigenfunction associated with $\mu_{j}$; that is, it satisfies $\left\|u_{j}\right\|_{L^{2}(\Omega)}=1$. It is well known that $\left\{\sqrt{\mu_{j}}\right\}_{j \geq 1}$ are exactly the real characteristic values of the operator-valued function $\omega \mapsto(1 / 2) I-\mathcal{K}_{\Omega}^{\omega}$. See 34. 
Let $\omega \notin\left\{\sqrt{\mu_{j}}\right\}_{j \geq 1}$. Introduce $N_{\Omega}^{\omega}(x, z)$ to be the Neumann function for $\Delta+\omega^{2}$ in $\Omega$ corresponding to a Dirac mass at $z$. That is, $N_{\Omega}^{\omega}$ is the unique solution to

$$
\begin{cases}\left(\Delta_{x}+\omega^{2}\right) N_{\Omega}^{\omega}(x, z)=-\delta_{z} & \text { in } \Omega \\ \left.\frac{\partial N_{\Omega}^{\omega}}{\partial \nu}\right|_{\partial \Omega}=0 & \text { on } \partial \Omega\end{cases}
$$

The following identity from [4, 6] relates the fundamental solution $\Gamma_{\omega}$ to the Neumann function $N_{\Omega}^{\omega}$ :

$$
-\left(\frac{1}{2} I-\mathcal{K}_{\Omega}^{\omega}\right)^{-1}\left(\Gamma_{\omega}(\cdot-z)\right)(x)=N_{\Omega}^{\omega}(x, z), \quad x \in \partial \Omega, z \in \Omega .
$$

The spectral decomposition,

$$
N_{\Omega}^{\omega}(x, z)=\sum_{j=1}^{+\infty} \frac{u_{j}(x) \overline{u_{j}}(z)}{\mu_{j}-\omega^{2}}
$$

will be of use to us. We refer the reader to [32, p. 246] for its proof.

Finally, we shall recall the concept of capacity. Suppose $d=2$ and let $\left(\varphi_{e}, a\right) \in$ $L^{2}(\partial D) \times \mathbb{R}$ denote the unique solution of the system

$$
\left\{\begin{array}{l}
\frac{1}{2 \pi} \int_{\partial D} \ln |x-y| \varphi_{e}(y) d \sigma(y)+a=0, \quad \text { on } \partial D, \\
\int_{\partial D} \varphi_{e}(y) d \sigma(y)=1 .
\end{array}\right.
$$

The logarithmic capacity of $\partial D$ is defined by

$$
\operatorname{cap}(\partial D):=e^{2 \pi a},
$$

where $a$ is given by (2.6).

If $d=3$, there exists a unique $\varphi_{e} \in L^{2}(\partial D)$ such that

$$
\left\{\begin{array}{l}
\int_{\partial D} \frac{\varphi_{e}(y)}{|x-y|} d \sigma(y)=\text { constant, } \quad \text { on } \partial D \\
\int_{\partial D} \varphi_{e}(y) d \sigma(y)=1
\end{array}\right.
$$

The capacity of $\partial D$ in three dimensions is defined to be

$$
\frac{1}{\operatorname{cap}(\partial D)}:=-\frac{1}{4 \pi} \int_{\partial D} \frac{1}{|x-y|} \varphi_{e}(y) d \sigma(y) \text {. }
$$

\section{Eigenvalue perturbations Caused by SMall PERFECTLY CONDUCTING INCLUSIONS}

Suppose that the inclusion $D$ is perfectly conducting. Let $0<\mu_{1}^{\epsilon} \leq \mu_{2}^{\epsilon} \leq \ldots$ be the eigenvalues of $-\Delta$ in $\Omega_{\epsilon}:=\Omega \backslash \bar{D}$ with the Neumann condition on $\partial \Omega$ and the Dirichlet condition on $\partial D$. We arrange them repeatedly according to their multiplicity.

Fix $j$ and suppose that the eigenvalue $\mu_{j}$ is simple. Note that this assumption is not essential in what follows, though its genericity is confirmed in [1, 35. It is made for ease of exposition. Then there exists a simple eigenvalue $\mu_{j}^{\epsilon}$ near $\mu_{j}$ 
associated to the normalized eigenfunction $u_{j}^{\epsilon}$; that is, $u_{j}^{\epsilon}$ satisfies the following problem:

$$
\begin{cases}\Delta u_{j}^{\epsilon}+\mu_{j}^{\epsilon} u_{j}^{\epsilon}=0 & \text { in } \Omega_{\epsilon}, \\ \frac{\partial u_{j}^{\epsilon}}{\partial \nu}=0 & \text { on } \partial \Omega, \\ u_{j}^{\epsilon}=0 & \text { on } \partial D .\end{cases}
$$

From [5], we know that the solution $u_{j}^{\epsilon}$ of (3.1) can be represented as

$$
u_{j}^{\epsilon}(x)=\mathcal{D}_{\Omega}^{\sqrt{\mu_{j}^{\epsilon}}}\left(\left.u_{j}^{\epsilon}\right|_{\partial \Omega}\right)(x)+\mathcal{S}_{D}^{\sqrt{\mu_{j}^{\epsilon}}}(\phi)(x), \quad x \in \Omega_{\epsilon},
$$

where $\psi:=\left.u_{j}^{\epsilon}\right|_{\partial \Omega} \in L^{2}(\partial \Omega)$ and $\phi \in L^{2}(\partial D)$ satisfy the following system of integral equations:

$$
\begin{cases}\left(\frac{1}{2} I-\mathcal{K}_{\Omega}^{\sqrt{\mu_{j}^{\epsilon}}}\right)(\psi)(x)-\mathcal{S}_{D}^{\sqrt{\mu_{j}^{\epsilon}}}(\phi)(x)=0, & x \in \partial \Omega, \\ \mathcal{D}_{\Omega}^{\sqrt{\mu_{j}^{\epsilon}}}(\psi)(x)+\mathcal{S}_{D}^{\sqrt{\mu_{j}^{\epsilon}}}(\phi)(x)=0, & x \in \partial D .\end{cases}
$$

Our strategy for deriving complete asymptotic expansions of the perturbations in the eigenvalues relies on expanding the operator-valued function

$$
\omega \mapsto\left(\begin{array}{cc}
\left(\frac{1}{2} I-\mathcal{K}_{\Omega}^{\omega}\right) & -\mathcal{S}_{D}^{\omega} \\
\mathcal{D}_{\Omega}^{\omega} & \mathcal{S}_{D}^{\omega}
\end{array}\right)
$$

in terms of $\epsilon$ and then, on calculating the asymptotic expressions of its characteristic values with the help of the generalized Rouché theorem.

3.1. Inclusions far away from the boundary. We assume that the inclusion $D$ is separated from the boundary. More precisely, we assume that there exists a constant $c_{0}>0$ such that $\operatorname{dist}(z, \partial \Omega) \geq 2 c_{0}>0$, that $\epsilon$, the order of magnitude of the diameter of the inclusion, is sufficiently small, and that the distance of the inclusion to $\mathbb{R}^{d} \backslash \bar{\Omega}$ is larger than $c_{0}$.

We need the following lemma.

Lemma 3.1. Let $\psi \in L^{2}(\partial \Omega)$ and let $\varphi \in L^{2}(\partial D)$. Define $\tilde{\varphi}(x)=\epsilon \varphi(\epsilon x+z)$, $x \in \partial B$. Then, for $x \in \partial B$, we have

$$
\begin{aligned}
& \mathcal{S}_{D}^{\omega}(\varphi)(\epsilon x+z) \\
& =\frac{1}{2 \pi} \sum_{n=0}^{+\infty}(-1)^{n} \frac{(\omega \epsilon)^{2 n}}{2^{2 n}(n !)^{2}} \int_{\partial B}|x-y|^{2 n}\left(\ln (\omega \epsilon|x-y|)+\ln \gamma-\sum_{j=1}^{n} \frac{1}{j}\right) \tilde{\varphi}(y) d \sigma(y),
\end{aligned}
$$

for $d=2$, where $2 \gamma=e^{\tilde{\gamma}-i \pi / 2}$ and $\tilde{\gamma}$ is Euler's constant, while for $d=3$,

$$
\mathcal{S}_{D}^{\omega}(\varphi)(\epsilon x+z)=-\frac{1}{4 \pi} \sum_{n=0}^{+\infty} \frac{1}{n !}(i \omega \epsilon)^{n} \int_{\partial B}|x-y|^{n-1} \tilde{\varphi}(y) d \sigma(y) .
$$

On the other hand, we have

$$
\mathcal{D}_{\Omega}^{\omega}(\psi)(\epsilon x+z)=\sum_{n=0}^{+\infty} \epsilon^{n} \sum_{|\alpha|=n} \frac{1}{\alpha !} \partial^{\alpha} \mathcal{D}_{\Omega}^{\omega}(\psi)(z) x^{\alpha}, \quad x \in \partial B, d=2,3,
$$


and for $d=2,3$,

$$
\mathcal{S}_{D}^{\omega}(\varphi)(x)=\sum_{n=0}^{+\infty}(-1)^{n} \epsilon^{n+d-2} \sum_{|\alpha|=n} \frac{1}{\alpha !} \partial^{\alpha} \Gamma_{\omega}(x-z)\left(\int_{\partial B} y^{\alpha} \tilde{\varphi}(y) d \sigma(y)\right), \quad x \in \partial \Omega .
$$

Proof. For any $\tilde{x}, \tilde{y} \in \partial D$, we have

$$
\mathcal{S}_{D}^{\omega}(\varphi)(\tilde{x})=\int_{\partial D} \Gamma_{\omega}(\tilde{x}-\tilde{y}) \varphi(\tilde{y}) d \sigma(\tilde{y}) .
$$

By the change of variables $\tilde{x}=\epsilon x+z$ and $\tilde{y}=\epsilon y+z$, we obtain that

$$
\begin{aligned}
\mathcal{S}_{D}^{\omega}(\varphi)(\tilde{x}) & =\epsilon^{d-1} \int_{\partial B} \Gamma_{\omega}(\epsilon(x-y)) \varphi(\epsilon y+z) d \sigma(y) \\
& =\epsilon^{d-2} \int_{\partial B} \Gamma_{\omega}(\epsilon(x-y)) \tilde{\varphi}(y) d \sigma(y) .
\end{aligned}
$$

The first two formulae immediately follow from the following Taylor expansion of $\Gamma_{\omega}(\epsilon x)$ as $\epsilon \rightarrow 0$ :

$$
\Gamma_{\omega}(\epsilon x)= \begin{cases}\frac{1}{2 \pi} \sum_{n=0}^{+\infty}(-1)^{n} \frac{(\omega \epsilon)^{2 n}}{2^{2 n}(n !)^{2}}|x|^{2 n}\left(\ln (\omega \epsilon|x|)+\ln \gamma-\sum_{j=1}^{n} \frac{1}{j}\right), & d=2, \\ -\frac{1}{4 \pi \epsilon} \sum_{n=0}^{+\infty} \frac{1}{n !}(i \omega \epsilon)^{n}|x|^{n-1}, & d=3 .\end{cases}
$$

Since $\left(\Delta+\omega^{2}\right) \mathcal{D}_{\Omega}^{\omega}(\psi)=0$ in $\Omega, \mathcal{D}_{\Omega}^{\omega}(\psi)$ is a smooth function in $\Omega$ and its Taylor expansion at $z$ yields

$$
\mathcal{D}_{\Omega}^{\omega}(\psi)(\epsilon x+z)=\sum_{n=0}^{+\infty} \epsilon^{n} \sum_{|\alpha|=n} \frac{1}{\alpha !} \partial^{\alpha} \mathcal{D}_{\Omega}^{\omega}(\psi)(z) x^{\alpha} .
$$

Finally, for any $x \in \partial \Omega$, it is easy to see that

$$
\begin{aligned}
\mathcal{S}_{D}^{\omega}(\varphi)(x) & =\int_{\partial D} \Gamma_{\omega}(x-\tilde{y}) \varphi(\tilde{y}) d \sigma(\tilde{y}) \\
& =\epsilon^{d-2} \int_{\partial B} \Gamma_{\omega}(x-z-\epsilon y) \tilde{\varphi}(y) d \sigma(y) \\
& =\epsilon^{d-2} \int_{\partial B} \sum_{n=0}^{+\infty}(-1)^{n} \epsilon^{n} \sum_{|\alpha|=n} \frac{1}{\alpha !} \partial^{\alpha} \Gamma_{\omega}(x-z) y^{\alpha} \tilde{\varphi}(y) d \sigma(y) \\
& =\sum_{n=0}^{+\infty}(-1)^{n} \epsilon^{n+d-2} \sum_{|\alpha|=n} \frac{1}{\alpha !} \partial^{\alpha} \Gamma_{\omega}(x-z) \int_{\partial B} y^{\alpha} \tilde{\varphi}(y) d \sigma(y),
\end{aligned}
$$

which completes the proof of the lemma. 
Suppose $d=2$. By Lemma 3.1 we have from (3.2) that (3.3)

$$
\left\{\begin{array}{l}
\left(\frac{1}{2} I-\mathcal{K}_{\Omega}^{\omega}\right)(\psi)(x) \\
-\sum_{n=0}^{+\infty}(-1)^{n} \epsilon^{n} \sum_{|\alpha|=n} \frac{1}{\alpha !} \partial^{\alpha} \Gamma_{\omega}(x-z)\left(\int_{\partial B} y^{\alpha} \tilde{\phi}(y) d \sigma(y)\right)=0, \quad x \in \partial \Omega, \\
\sum_{n=0}^{+\infty} \epsilon^{n} \sum_{|\alpha|=n} \frac{1}{\alpha !} \partial^{\alpha} \mathcal{D}_{\Omega}^{\omega}(\psi)(z) x^{\alpha}+\frac{1}{2 \pi} \sum_{n=0}^{+\infty}(-1)^{n} \frac{(\omega \epsilon)^{2 n}}{2^{2 n}(n !)^{2}} \\
\times \int_{\partial B}|x-y|^{2 n}\left(\ln (\omega \epsilon|x-y|)+\ln \gamma-\sum_{j=1}^{n} \frac{1}{j}\right) \tilde{\phi}(y) d \sigma(y)=0, \quad x \in \partial B,
\end{array}\right.
$$

where $\omega=\sqrt{\mu_{j}^{\epsilon}}, \psi:=\left.u_{j}^{\epsilon}\right|_{\partial \Omega}$, and $\tilde{\phi}(x):=\epsilon \phi(\epsilon x+z), x \in \partial B$.

If $d=3$, then

$$
\left\{\begin{array}{l}
\left(\frac{1}{2} I-\mathcal{K}_{\Omega}^{\omega}\right)(\psi)(x) \\
-\sum_{n=0}^{+\infty}(-1)^{n} \epsilon^{n+1} \sum_{|\alpha|=n} \frac{1}{\alpha !} \partial^{\alpha} \Gamma_{\omega}(x-z)\left(\int_{\partial B} y^{\alpha} \tilde{\phi}(y) d \sigma(y)\right)=0, \quad x \in \partial \Omega \\
\sum_{n=0}^{+\infty} \epsilon^{n} \sum_{|\alpha|=n} \frac{1}{\alpha !} \partial^{\alpha} \mathcal{D}_{\Omega}^{\omega}(\psi)(z) x^{\alpha} \\
-\frac{1}{4 \pi} \sum_{n=0}^{+\infty} \frac{1}{n !}(i \omega \epsilon)^{n} \int_{\partial B}|x-y|^{n-1} \tilde{\phi}(y) d \sigma(y)=0, \quad x \in \partial B .
\end{array}\right.
$$

The systems of integral equations (3.3) and (3.4) may alternatively be written in the form

$$
\mathcal{A}_{\epsilon}^{d}(\omega)\left(\begin{array}{c}
\psi \\
\tilde{\phi}
\end{array}\right)=0
$$

where

$$
\mathcal{A}_{\epsilon}^{3}(\omega)=\sum_{n=0}^{+\infty}(\omega \epsilon)^{n} \mathcal{A}_{n}^{3}(\omega)
$$

with

$$
\mathcal{A}_{0}^{3}(\omega):=\left(\begin{array}{cc}
\frac{1}{2} I-\mathcal{K}_{\Omega}^{\omega} & 0 \\
\mathcal{D}_{\Omega}^{\omega}(\cdot)(z) & -\frac{1}{4 \pi} \int_{\partial B}|x-y|^{-1} \cdot d \sigma(y)
\end{array}\right),
$$

and for $n \geq 1$,

$$
\mathcal{A}_{n}^{3}(\omega):=\left(\begin{array}{cc}
0 & \frac{1}{\omega} A_{n-1} \\
B_{n} & -\frac{1}{4 \pi} \frac{i^{n}}{n !} \int_{\partial B}|x-y|^{n-1} \cdot d \sigma(y)
\end{array}\right),
$$


where

$$
\begin{aligned}
& A_{n}:=\frac{(-1)^{n+1}}{\omega^{n}} \sum_{|\alpha|=n} \frac{1}{\alpha !} \partial^{\alpha} \Gamma_{\omega}(x-z)\left(\int_{\partial B} y^{\alpha} \cdot d \sigma(y)\right), \\
& B_{n}:=\frac{1}{\omega^{n}} \sum_{|\alpha|=n} \frac{1}{\alpha !} \partial^{\alpha} \mathcal{D}_{\Omega}^{\omega}(\cdot)(z) x^{\alpha}
\end{aligned}
$$

and $A_{0}:=-\Gamma_{\omega}(x-z)\left(\int_{\partial B} \cdot d \sigma(y)\right)$.

In the two-dimensional case,

$$
\mathcal{A}_{\epsilon}^{2}(\omega)=\sum_{n=0}^{+\infty}(\omega \epsilon)^{n}\left(\mathcal{A}_{n}^{2}(\omega)+\ln (\omega \epsilon) \mathcal{B}_{n}^{2}(\omega)\right)
$$

with

$$
\begin{aligned}
\mathcal{A}_{0}^{2}(\omega) & :=\left(\begin{array}{cc}
\frac{1}{2} I-\mathcal{K}_{\Omega}^{\omega} & -\Gamma_{\omega}(x-z)\left(\int_{\partial B} \cdot d \sigma(y)\right) \\
\mathcal{D}_{\Omega}^{\omega}(\cdot)(z) & \frac{1}{2 \pi} \int_{\partial B}(\ln |x-y|+\ln \gamma) \cdot d \sigma(y)
\end{array}\right), \\
\mathcal{B}_{0}^{2}(\omega) & :=\left(\begin{array}{ll}
0 & 0 \\
0 & \frac{1}{2 \pi} \int_{\partial B} \cdot d \sigma(y)
\end{array}\right),
\end{aligned}
$$

and, for $n \geq 1$,

$$
\mathcal{A}_{2 n}^{2}(\omega):=\left(\begin{array}{cc}
0 & A_{2 n} \\
B_{2 n} & C_{2 n}
\end{array}\right), \quad \mathcal{A}_{2 n-1}^{2}(\omega):=\left(\begin{array}{cc}
0 & A_{2 n-1} \\
B_{2 n-1} & 0
\end{array}\right),
$$

and

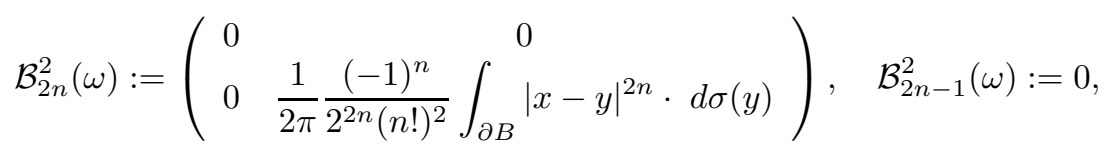

where $A_{n}$ and $B_{n}$ are as above and

$$
C_{2 n}:=\frac{1}{2 \pi} \frac{(-1)^{n}}{2^{2 n}(n !)^{2}} \int_{\partial B}|x-y|^{2 n}\left(\ln |x-y|+\ln \gamma-\sum_{j=1}^{n} \frac{1}{j}\right) \cdot d \sigma(y) .
$$

It is therefore obvious that the pair of functions $(\psi, \tilde{\phi})$ is then a characteristic function of the integral operator-valued function $\mathcal{A}_{\epsilon}^{d}, d=2,3$, associated with the characteristic value $\sqrt{\mu_{j}^{\epsilon}}$. We give a rigorous study of the integral operator-valued function $\omega \mapsto \mathcal{A}_{\epsilon}^{d}(\omega)$, when $\omega$ is in a small complex neighborhood of $\sqrt{\mu_{j}}$. We proceed from the generalized Rouché theorem to construct the complete asymptotic expansions for $\mu_{j}^{\epsilon}$.

At this stage, we emphasize the fact that the asymptotic parameter is in fact $\omega \epsilon$ and not $\epsilon$, which shows that the asymptotic expansions of $\mu_{j}^{\epsilon}-\mu_{j}$ we are going to derive are not only valid for fixed $j$ when $\epsilon$ goes to zero but also uniformly for the set of first $j$ eigenvalues such that the $\sqrt{\mu_{j}} \epsilon$ remain small. 
In order to simplify notation we introduce the single layer potential associated with the Laplacian:

$$
\mathcal{S}_{B}^{0} \phi(x)= \begin{cases}\frac{1}{2 \pi} \int_{\partial B} \ln |x-y| \phi(y) d \sigma(y) & \text { if } d=2, \\ -\frac{1}{4 \pi} \int_{\partial B} \frac{1}{|x-y|} \phi(y) d \sigma(y) & \text { if } d=3 .\end{cases}
$$

It is easy to see that the $\left\{\sqrt{\mu_{j}^{\epsilon}}\right\}_{j \geq 1}$ are exactly the real characteristic values of the operator-valued function $\mathcal{A}_{\epsilon}^{d}$, for $0 \leq \epsilon \leq \epsilon_{0}, \epsilon_{0}>0$. Conversely, if $\omega$ is a real characteristic value of the operator-valued function $\mathcal{A}_{\epsilon}^{d}$, then $\omega^{2}$ is an eigenvalue of (3.1).

The next three lemmas can be proved by slightly modifying the arguments given in 14 .

Lemma 3.2. The operator-valued function $\mathcal{A}_{\epsilon}^{d}(\omega)$ is Fredholm analytic with index 0 in $\mathbb{C} \backslash i \mathbb{R}^{-}$and $\left(\mathcal{A}_{\epsilon}^{d}\right)^{-1}(\omega)$ is a meromorphic function. If $\omega$ is a real characteristic value of the operator-valued function $\mathcal{A}_{\epsilon}^{d}$ (or equivalently, a real pole of $\left(\mathcal{A}_{\epsilon}^{d}\right)^{-1}(\omega)$ ), then there exists $j$ such that $\omega=\sqrt{\mu_{j}^{\epsilon}}$.

Lemma 3.3. Any $\sqrt{\mu_{j}}$ is a simple pole of the operator-valued function $\left(\mathcal{A}_{0}^{d}\right)^{-1}(\omega)$.

Lemma 3.4. Let $\omega_{0}=\sqrt{\mu_{j}}$ and suppose that $\mu_{j}$ is simple. Then there exists a positive constant $\delta_{0}$ such that for $|\delta|<\delta_{0}$, the operator-valued function $\omega \mapsto \mathcal{A}_{\epsilon}^{d}(\omega)$ has exactly one characteristic value in $\overline{V_{\delta_{0}}}\left(\omega_{0}\right)$, where $V_{\delta_{0}}\left(\omega_{0}\right)$ is a disc of center $\omega_{0}$ and radius $\delta_{0}>0$. This characteristic value is analytic with respect to $\epsilon$ in ]$-\epsilon_{0}, \epsilon_{0}[$. Moreover, the following assertions hold:

$$
\left\{\begin{array}{l}
\mathcal{M}\left(\mathcal{A}_{\epsilon}^{d}(\omega) ; \partial V_{\delta_{0}}\right)=1 \\
\left(\mathcal{A}_{\epsilon}^{d}\right)^{-1}(\omega)=\left(\omega-\omega_{0}\right)^{-1} \mathcal{L}_{\epsilon}^{d}+\mathcal{R}_{\epsilon}^{d}(\omega) \\
\mathcal{L}_{\epsilon}^{d}: \operatorname{Ker}\left(\left(\mathcal{A}_{\epsilon}^{d}\left(\omega_{\epsilon}\right)\right)^{*}\right) \rightarrow \operatorname{Ker}\left(\mathcal{A}_{\epsilon}^{d}\left(\omega_{\epsilon}\right)\right)
\end{array}\right.
$$

where $\mathcal{R}_{\epsilon}^{d}(\omega)$ is a holomorphic function with respect to $\left.(\epsilon, \omega) \in\right]-\epsilon_{0}, \epsilon_{0}\left[\times V_{\delta_{0}}\left(\omega_{0}\right)\right.$ and $\mathcal{L}_{\epsilon}^{d}$ is a finite-dimensional operator.

Based on the generalized Rouché theorem, we are now ready to derive complete asymptotic formulae for the characteristic values of $\omega \mapsto \mathcal{A}_{\epsilon}^{d}(\omega)$. Applying Theorem 2.5. we get the following lemma.

Lemma 3.5. Let $\omega_{0}=\sqrt{\mu_{j}}$ and suppose that $\mu_{j}$ is simple. Then $\omega_{\epsilon}=\sqrt{\mu_{j}^{\epsilon}}$ is given by

$$
\omega_{\epsilon}-\omega_{0}=\frac{1}{2 i \pi} \operatorname{tr} \int_{\partial V_{\delta_{0}}}\left(\omega-\omega_{0}\right)\left(\mathcal{A}_{\epsilon}^{d}\right)^{-1}(\omega) \frac{d}{d \omega} \mathcal{A}_{\epsilon}^{d}(\omega) d \omega .
$$

Following once again [14, we obtain the following complete asymptotic expansion for the eigenvalue perturbations in the three-dimensional case.

Theorem 3.6. Suppose $d=3$. Then the following asymptotic expansion holds:

$$
\omega_{\epsilon}-\omega_{0}=\frac{1}{2 i \pi} \sum_{p=1}^{+\infty} \frac{1}{p} \sum_{n=p}^{+\infty} \epsilon^{n} \operatorname{tr} \int_{\partial V_{\delta_{0}}} B_{n, p}^{3}(\omega) d \omega
$$

where

$$
B_{n, p}^{3}(\omega)=(-1)^{p} \sum_{\substack{n_{1}+\ldots+n_{p}=n \\ n_{i} \geq 1}}\left(\mathcal{A}_{0}^{3}\right)^{-1}(\omega) \mathcal{A}_{n_{1}}^{3}(\omega) \ldots\left(\mathcal{A}_{0}^{3}\right)^{-1}(\omega) \mathcal{A}_{n_{p}}^{3}(\omega) \omega^{n} .
$$


Proof. If $\epsilon$ is small enough, then the following Neumann series converges uniformly with respect to $\omega$ in $\partial V_{\delta_{0}}$ :

$$
\left(\mathcal{A}_{\epsilon}^{3}\right)^{-1}(\omega)=\sum_{p=0}^{\infty}\left[\left(\mathcal{A}_{0}^{3}\right)^{-1}(\omega)\left(\mathcal{A}_{0}^{3}(\omega)-\mathcal{A}_{\epsilon}^{3}(\omega)\right)\right]^{p}\left(\mathcal{A}_{0}^{3}\right)^{-1}(\omega)
$$

and hence we may deduce that

$\omega_{\epsilon}-\omega_{0}=\frac{1}{2 i \pi} \sum_{p=0}^{\infty} \operatorname{tr} \int_{\partial V_{\delta_{0}}}\left(\omega-\omega_{0}\right)\left[\left(\mathcal{A}_{0}^{3}\right)^{-1}(\omega)\left(\mathcal{A}_{0}^{3}(\omega)-\mathcal{A}_{\epsilon}^{3}(\omega)\right)\right]^{p}\left(\mathcal{A}_{0}^{3}\right)^{-1}(\omega) \frac{d}{d \omega} \mathcal{A}_{\epsilon}^{3}(\omega) d \omega$.

By using the property (2.1) of the trace together with the identity

$$
\frac{d}{d \omega}\left(\mathcal{A}_{0}^{3}\right)^{-1}(\omega)=-\left(\mathcal{A}_{0}^{3}\right)^{-1}(\omega) \frac{d \mathcal{A}_{0}^{3}}{d \omega}(\omega)\left(\mathcal{A}_{0}^{3}\right)^{-1}(\omega)
$$

we have

$$
\begin{aligned}
& \operatorname{tr} \int_{\partial V_{\delta_{0}}}\left(\omega-\omega_{0}\right) \frac{1}{p} \frac{d}{d \omega}\left[\left(\mathcal{A}_{0}^{3}\right)^{-1}(\omega)\left(\mathcal{A}_{0}^{3}(\omega)-\mathcal{A}_{\epsilon}^{3}(\omega)\right)\right]^{p} d \omega \\
& =\operatorname{tr} \int_{\partial V_{\delta_{0}}}\left(\omega-\omega_{0}\right)\left[\left(\mathcal{A}_{0}^{3}\right)^{-1}(\omega)\left(\mathcal{A}_{0}^{3}(\omega)-\mathcal{A}_{\epsilon}^{3}(\omega)\right)\right]^{p-1}\left(\mathcal{A}_{0}^{3}\right)^{-1}(\omega) \frac{d}{d \omega}\left(\mathcal{A}_{0}^{3}(\omega)-\mathcal{A}_{\epsilon}^{3}(\omega)\right) d \omega \\
& -\operatorname{tr} \int_{\partial V_{\delta_{0}}}\left(\omega-\omega_{0}\right)\left[\left(\mathcal{A}_{0}^{3}\right)^{-1}(\omega)\left(\mathcal{A}_{0}^{3}(\omega)-\mathcal{A}_{\epsilon}^{3}(\omega)\right)\right]^{p}\left(\mathcal{A}_{0}^{3}\right)^{-1}(\omega) \frac{d}{d \omega} \mathcal{A}_{0}^{3}(\omega) d \omega,
\end{aligned}
$$

and therefore,

$$
\begin{gathered}
\omega_{\epsilon}-\omega_{0}=-\frac{1}{2 i \pi} \sum_{p=1}^{\infty} \operatorname{tr} \int_{\partial V_{\delta_{0}}}\left(\omega-\omega_{0}\right) \frac{1}{p} \frac{d}{d \omega}\left[\left(\mathcal{A}_{0}^{3}\right)^{-1}(\omega)\left(\mathcal{A}_{0}^{3}(\omega)-\mathcal{A}_{\epsilon}^{3}(\omega)\right)\right]^{p} d \omega \\
+\frac{1}{2 i \pi} \operatorname{tr} \int_{\partial V_{\delta_{0}}}\left(\omega-\omega_{0}\right)\left(\mathcal{A}_{0}^{3}\right)^{-1}(\omega) \frac{d}{d \omega} \mathcal{A}_{0}^{3}(\omega) d \omega .
\end{gathered}
$$

Because of Lemma 3.3, $\omega_{0}$ is a simple pole of $\left(\mathcal{A}_{0}^{3}\right)^{-1}(\omega)$ and $\mathcal{A}_{0}^{3}(\omega)$ is analytic, and hence we get

$$
\int_{\partial V_{\delta_{0}}}\left(\omega-\omega_{0}\right)\left(\mathcal{A}_{0}^{3}\right)^{-1}(\omega) \frac{d}{d \omega} \mathcal{A}_{0}^{3}(\omega) d \omega=0 .
$$

Thus, it follows that

$$
\omega_{\epsilon}-\omega_{0}=-\frac{1}{2 i \pi} \sum_{p=1}^{\infty} \operatorname{tr} \int_{\partial V_{\delta_{0}}}\left(\omega-\omega_{0}\right) \frac{1}{p} \frac{d}{d \omega}\left[\left(\mathcal{A}_{0}^{3}\right)^{-1}(\omega)\left(\mathcal{A}_{0}^{3}(\omega)-\mathcal{A}_{\epsilon}^{3}(\omega)\right)\right]^{p} d \omega .
$$

Now, a simple integration by parts yields

$$
\omega_{\epsilon}-\omega_{0}=\frac{1}{2 i \pi} \sum_{p=1}^{\infty} \frac{1}{p} \operatorname{tr} \int_{\partial V_{\delta_{0}}}\left(\left(\mathcal{A}_{0}^{3}\right)^{-1}(\omega)\left(\mathcal{A}_{0}^{3}(\omega)-\mathcal{A}_{\epsilon}^{3}(\omega)\right)\right)^{p} d \omega
$$

Notice from (3.5) that

$$
\begin{aligned}
& \left(\left(\mathcal{A}_{0}^{3}\right)^{-1}(\omega)\left(\mathcal{A}_{0}^{3}(\omega)-\mathcal{A}_{\epsilon}^{3}(\omega)\right)\right)^{p} \\
& \quad=(-1)^{p} \sum_{n=p}^{\infty} \epsilon^{n} \sum_{\substack{n_{1}+\ldots+n_{p}=n \\
n_{i} \geq 1}}\left(\mathcal{A}_{0}^{3}\right)^{-1}(\omega) \mathcal{A}_{n_{1}}^{3}(\omega) \ldots\left(\mathcal{A}_{0}^{3}\right)^{-1}(\omega) \mathcal{A}_{n_{p}}^{3}(\omega) \omega^{n} .
\end{aligned}
$$

Therefore, upon inserting this into the latter formula we arrive at the desired asymptotic expansion. 
The following theorem can be proved in the same way as Theorem 3.6 .

Theorem 3.7. Suppose $d=2$. Then the following asymptotic expansion holds:

$$
\omega_{\epsilon}-\omega_{0}=\frac{1}{2 i \pi} \sum_{p=1}^{+\infty} \frac{1}{p} \sum_{n=p}^{+\infty} \epsilon^{n} \operatorname{tr} \int_{\partial V_{\delta_{0}}} B_{n, p}^{2}(\omega) d \omega
$$

where

$$
\begin{gathered}
B_{n, p}^{2}(\omega)=(-1)^{p} \sum_{\substack{n_{1}+\ldots+n_{p}=n \\
n_{i} \geq 1}}\left(\mathcal{A}_{0}^{2}(\omega)+\ln (\omega \epsilon) \mathcal{B}_{0}^{2}(\omega)\right)^{-1}\left(\mathcal{A}_{n_{1}}^{2}(\omega)+\ln (\omega \epsilon) \mathcal{B}_{n_{1}}^{2}(\omega)\right) \\
\ldots\left(\mathcal{A}_{0}^{2}(\omega)+\ln (\omega \epsilon) \mathcal{B}_{0}^{2}(\omega)\right)^{-1}\left(\mathcal{A}_{n_{p}}^{2}(\omega)+\ln (\omega \epsilon) \mathcal{B}_{n_{p}}^{2}(\omega)\right) \omega^{n} .
\end{gathered}
$$

As a simplest case, let us now find the leading-order term in the asymptotic expansion of $\mu_{j}^{\epsilon}-\mu_{j}$ as $\epsilon \rightarrow 0$.

Suppose $d=3$. Recall from (3.6) that

$$
\omega_{\epsilon}-\omega_{0}=-\frac{\epsilon}{2 \pi i} \operatorname{tr} \int_{\partial V_{\delta_{0}}}\left(\mathcal{A}_{0}^{3}\right)^{-1}(\omega) \mathcal{A}_{1}^{3}(\omega) \omega d \omega .
$$

Let the space $W_{1}^{2}(\partial B)$ be the set of functions $f \in L^{2}(\partial B)$ such that $\partial f / \partial T \in$ $L^{2}(\partial B)$, where $\partial / \partial T$ denotes the tangential derivative on $\partial B$. It is proved in 36 ] that $\mathcal{S}_{B}^{0}: L^{2}(\partial B) \rightarrow W_{1}^{2}(\partial B)$ is invertible. Therefore, we have

$$
\left(\mathcal{S}_{B}^{0}\right)^{-1}(1)=\operatorname{cap}(\partial B) \varphi_{e}
$$

where $\operatorname{cap}(\partial B)$ and $\varphi_{e}$ are defined in (2.7) and (2.8). It is now easy to see that

$$
\left(\mathcal{A}_{0}^{3}\right)^{-1}(\omega)=\left(\begin{array}{cc}
\left(\frac{1}{2} I-\mathcal{K}_{\Omega}^{\omega}\right)^{-1} & 0 \\
-C \mathcal{D}_{\Omega}^{\omega}\left(\frac{1}{2} I-\mathcal{K}_{\Omega}^{\omega}\right)^{-1}(\cdot)(z) \varphi_{e} & \left(\mathcal{S}_{B}^{0}\right)^{-1}
\end{array}\right),
$$

where $C:=\operatorname{cap}(\partial B)$. It then follows from (2.4) that

$$
\begin{aligned}
& \left(\mathcal{A}_{0}^{3}\right)^{-1}(\omega) \mathcal{A}_{1}^{3}(\omega)= \\
& \left(\begin{array}{lr}
0 & \frac{1}{\omega} N_{\Omega}^{\omega}(x, z) \int_{\partial B} \cdot d \sigma(y) \\
\left(\mathcal{S}_{B}^{0}\right)^{-1}\left(\nabla \mathcal{D}_{\Omega}^{\omega}(\cdot)(z) \cdot x\right) & -C \mathcal{D}_{\Omega}^{\omega}\left(N_{\Omega}^{\omega}(\cdot, z)\right)(z) \varphi_{e} \int_{\partial B} \cdot d \sigma(y)-\frac{i \omega}{4 \pi} C \varphi_{e} \int_{\partial B} \cdot d \sigma(y)
\end{array}\right) .
\end{aligned}
$$

Since $\int_{\partial B} \varphi_{e} d \sigma=1$, we now have

$$
\begin{aligned}
\frac{1}{2 \pi i} \operatorname{tr} \int_{\partial V_{\delta_{0}}}\left(\mathcal{A}_{0}^{3}\right)^{-1}(\omega) \mathcal{A}_{1}^{3}(\omega) \omega d \omega & =-\frac{C}{2 \pi i} \operatorname{tr} \int_{\partial V_{\delta_{0}}} \mathcal{D}_{\Omega}^{\omega}\left(N_{\Omega}^{\omega}(\cdot, z)\right)(z) \varphi_{e} \int_{\partial B} \cdot d \sigma(y) d \omega \\
& =-\frac{C}{2 \pi i} \int_{\partial V_{\delta_{0}}} \mathcal{D}_{\Omega}^{\omega}\left(N_{\Omega}^{\omega}(\cdot, z)\right)(z) d \omega
\end{aligned}
$$

Since $-\Delta u_{j}=\mu_{j} u_{j}$ in $\Omega$ and $\frac{\partial u_{j}}{\partial \nu}=0$ on $\partial \Omega$, we have

$$
\mathcal{D}_{\Omega}^{\omega}\left(u_{j}\right)(z)=u_{j}(z)+\left(\mu_{j}-\omega^{2}\right) \int_{\Omega} \Gamma_{\omega}(z-y) u_{j}(y) d y .
$$

Since $\Gamma_{\omega}$ is analytic in $\omega \in V_{\delta_{0}}\left(\sqrt{\mu_{j}}\right)$ and $-\sqrt{\mu_{l}} \notin V_{\delta_{0}}\left(\sqrt{\mu_{j}}\right), \forall l \neq j$, if $\delta_{0}$ is sufficiently small, the spectral decomposition (2.5) and the residue theorem yield that

$$
\frac{1}{2 \pi i} \int_{\partial V_{\delta_{0}}} \mathcal{D}_{\Omega}^{\omega}\left(N_{\Omega}^{\omega}(\cdot, z)\right)(z) d \omega=\frac{1}{2 \pi i}\left|u_{j}(z)\right|^{2} \int_{\partial V_{\delta_{0}}} \frac{1}{\mu_{j}-\omega^{2}} d \omega=-\frac{1}{2 \sqrt{\mu_{j}}}\left|u_{j}(z)\right|^{2} .
$$


We thus get

$$
\frac{1}{2 i \pi} \operatorname{tr} \int_{\partial V_{\delta_{0}}}\left(\mathcal{A}_{0}^{3}\right)^{-1}(\omega) \mathcal{A}_{1}^{3}(\omega) \omega d \omega=\frac{1}{2 \sqrt{\mu_{j}}}\left|u_{j}(z)\right|^{2} \operatorname{cap}(\partial B),
$$

which yields the following corollary.

Corollary 3.8. Suppose $d=3$. Then the following asymptotic expansion holds:

$$
\mu_{j}^{\epsilon}-\mu_{j}=\epsilon \operatorname{cap}(\partial B)\left|u_{j}(z)\right|^{2}+O\left(\epsilon^{2}\right) .
$$

In particular, if $B$ is the unit ball, then $\operatorname{cap}(\partial B)=-4 \pi$ and (3.7) yields the formula obtained by Ozawa in [26]. It should be mentioned that Ozawa also obtained the $\epsilon^{2}$ term of the asymptotic expansions of $\mu_{j}^{\epsilon}-\mu_{j}$ when the inclusion is a sphere in [28. The second order term when the inclusion is of general shape can be explicitly computed using (3.6).

Let us now consider the two-dimensional case. Recall that

$$
\mathcal{A}_{0}^{2}(\omega)+\ln (\omega \epsilon) \mathcal{B}_{0}^{2}(\omega)=\left(\begin{array}{cc}
\frac{1}{2} I-\mathcal{K}_{\Omega}^{\omega} & -\Gamma_{\omega}(x-z)\left(\int_{\partial B} \cdot d \sigma(y)\right) \\
\mathcal{D}_{\Omega}^{\omega}(\cdot)(z) & \mathcal{S}_{B}^{0}+\frac{1}{2 \pi} \int_{\partial B}(\ln (\gamma \omega \epsilon)) \cdot d \sigma(y)
\end{array}\right) .
$$

Let $a$ and $\varphi_{e}$ be as defined by (2.8), and let $W_{0}$ be the collection of all functions $\psi \in W_{1}^{2}(\partial B)$ such that $\int_{\partial B} \varphi_{e} \psi d \sigma=0$. Then it is proved in [36] that $\mathcal{S}_{B}^{0}$ : $L_{0}^{2}(\partial B) \rightarrow W_{0}$ is invertible. Let $\left(\mathcal{S}_{B}^{0}\right)^{-1}$ denote its inverse. Then one can easily see that

$$
\left(\mathcal{A}_{0}^{2}(\omega)+\ln (\omega \epsilon) \mathcal{B}_{0}^{2}(\omega)\right)^{-1}\left(\begin{array}{c}
f \\
g
\end{array}\right)=\left(\begin{array}{c}
\left(\frac{1}{2} I-\mathcal{K}_{\Omega}^{\omega}\right)^{-1}(f)-C(f, g) N_{\Omega}^{\omega}(x, z) \\
C(f, g) \varphi_{e}+\left(\mathcal{S}_{B}^{0}\right)^{-1}(g-\bar{g})
\end{array}\right),
$$

where $\bar{g}=\int_{\partial B} g \varphi_{e} d \sigma$ and the constant $C(f, g)$ is defined to be

$$
C(f, g)=\frac{\bar{g}-\mathcal{D}_{\Omega}^{\omega}\left(\frac{1}{2} I-\mathcal{K}_{\Omega}^{\omega}\right)^{-1}(f)(z)}{\frac{\ln \gamma \omega \epsilon}{2 \pi}-\mathcal{D}_{\Omega}^{\omega}\left(N_{\Omega}^{\omega}(\cdot, z)\right)(z)-a} .
$$

Now, in exactly the same manner as in Corollary 3.8, we obtain from Theorem 3.7 that the leading-order term in the asymptotic expansion of $\mu_{j}^{\epsilon}-\mu_{j}$ in two dimensions is as follows.

Corollary 3.9. Suppose $d=2$. Then the following asymptotic expansion holds:

$$
\mu_{j}^{\epsilon}-\mu_{j}=-\frac{2 \pi}{\ln \left(\frac{\epsilon \sqrt{\mu_{j}}}{\operatorname{cap}(\partial B)}\right)}\left|u_{j}(z)\right|^{2}+o\left(\frac{1}{\ln (\epsilon)}\right) .
$$

In particular, since the logarithmic capacity of the unit disk $\operatorname{cap}(\partial B)=1$, (3.8) yields the formula derived by Ozawa in [27. See also Besson [15].

3.2. Inclusions nearly touching the boundary. In this section we study the eigenvalue problem in the presence of a diametrically small perfectly conducting inclusion that is nearly touching the boundary. Consider a small perfectly conducting inclusion $D$ inside $\Omega$ that is nearly touching the boundary $\partial \Omega$. We assume that $\partial \Omega$ is of class $\mathcal{C}^{2}$ and $D=\epsilon B+z$, where $z \in \Omega$ is such that $\operatorname{dist}(z, \partial \Omega)=\delta \epsilon$. Here $B$ is a bounded domain in $\mathbb{R}^{2}$ containing the origin with a connected $\mathcal{C}^{2}$-boundary and the constant $\delta>\max _{x \in \partial B}|x|$. We show that the leading-order term in the asymptotic expansion of the eigenvalue perturbations is the same as in Corollary 3.9 . 
The following lemma from [2] is of use to us.

Lemma 3.10. Suppose that $\partial \Omega$ is of class $\mathcal{C}^{2}$ and let $\psi \in \mathcal{C}^{0}(\partial \Omega)$. Let $z_{0}$ be the normal projection of $z$ onto $\partial \Omega$. Then, for any $x \in \partial B$,

$$
\int_{\partial \Omega} \frac{\epsilon}{\left|y-z_{0}-\epsilon\left(x-\delta \nu_{z_{0}}\right)\right|^{2}} \psi(y) d \sigma(y) \rightarrow \frac{\pi}{\left(\delta \nu_{z_{0}}-x\right) \cdot \nu_{z_{0}}} \psi\left(z_{0}\right) .
$$

Using Lemma 3.10 we prove the following.

Lemma 3.11. Suppose that $\partial \Omega$ is of class $\mathcal{C}^{2}$ and let $\psi \in \mathcal{C}^{0}(\partial \Omega)$. Let $z_{0}$ be the normal projection of $z$ onto $\partial \Omega$. For any $x \in \partial B$, we have

$$
\mathcal{D}_{\Omega}^{\omega}(\psi)(\epsilon x+z)=\left(\frac{1}{2} I+\mathcal{K}_{\Omega}^{\omega}\right)(\psi)\left(z_{0}\right)+o(1) \quad \text { as } \epsilon \rightarrow 0,
$$

where the remainder $o(1)$ is uniform in $x \in \partial B$.

Proof. Let $\nu_{z_{0}}$ denote the outward unit normal to $\partial \Omega$ at $z_{0}$. Since $z=z_{0}+z-z_{0}=$ $z_{0}-\delta \epsilon \nu_{z_{0}}$, for any $x \in \partial B$, we have

$$
\epsilon x+z=\epsilon x+z_{0}-\delta \epsilon \nu_{z_{0}}=z_{0}+\epsilon\left(x-\delta \nu_{z_{0}}\right) .
$$

Hence, we obtain

$$
\begin{aligned}
\mathcal{D}_{\Omega}^{\omega}(\psi)\left(z_{0}+\epsilon\left(x-\delta \nu_{z_{0}}\right)\right) \\
=-\frac{i \omega}{4} \int_{\partial \Omega}\left(H_{0}^{(1)^{\prime}}\left(\omega\left|y-z_{0}-\epsilon\left(x-\delta \nu_{z_{0}}\right)\right|\right)\left|y-z_{0}-\epsilon\left(x-\delta \nu_{z_{0}}\right)\right|\right) \\
\quad \times \frac{\left\langle y-z_{0}, \nu_{y}\right\rangle-\epsilon\left\langle x-\delta \nu_{z_{0}}, \nu_{y}\right\rangle}{\left|y-z_{0}-\epsilon\left(x-\delta \nu_{z_{0}}\right)\right|^{2}} \psi(y) d \sigma(y) .
\end{aligned}
$$

Since

$$
-\frac{i \omega}{4} H_{0}^{(1)^{\prime}}\left(\omega\left|t-z_{0}\right|\right)\left|t-z_{0}\right|=\frac{1}{2 \pi}-\frac{\omega^{2}}{4 \pi}\left|t-z_{0}\right|^{2} \ln \left|t-z_{0}\right|-\frac{\omega^{2}}{4 \pi}\left|t-z_{0}\right|^{2}\left(\ln (\omega \gamma)-\frac{1}{2}\right)+\ldots,
$$

as $t \rightarrow z_{0}$, we see that

$$
\begin{aligned}
& H_{0}^{(1)^{\prime}}\left(\omega\left|z_{0}-y+\epsilon\left(x-\delta \nu_{z_{0}}\right)\right|\right)\left|y-z_{0}-\epsilon\left(x-\delta \nu_{z_{0}}\right)\right| \\
& =H_{0}^{(1)^{\prime}}\left(\omega\left|y-z_{0}\right|\right)\left|y-z_{0}\right|+O(\epsilon)
\end{aligned}
$$

as $\epsilon$ tends to 0 .

Now, since $\partial \Omega$ is of class $\mathcal{C}^{2}$, we have (see [2])

$$
\frac{\left|\left\langle y-z, \nu_{y}\right\rangle\right|}{|y-z|^{2}}=O(1)
$$

and hence

which gives

$$
\left|\frac{\left\langle y-z_{0}, \nu_{y}\right\rangle-\epsilon\left\langle x-\delta \nu_{z_{0}}, \nu_{y}\right\rangle}{\left|y-z_{0}-\epsilon\left(x-\delta \nu_{z_{0}}\right)\right|^{2}}\right|=O(1)
$$

$$
\begin{aligned}
- & \frac{i \omega}{4} \int_{\partial \Omega}\left(H_{0}^{(1)^{\prime}}\left(\omega\left|y-z_{0}-\epsilon\left(x-\delta \nu_{z_{0}}\right)\right|\right)\left|y-z_{0}-\epsilon\left(x-\delta \nu_{z_{0}}\right)\right|\right) \\
& \times \frac{\left\langle y-z_{0}, \nu_{y}\right\rangle-\epsilon\left\langle x-\delta \nu_{z_{0}}, \nu_{y}\right\rangle}{\left|y-z_{0}-\epsilon\left(x-\delta \nu_{z_{0}}\right)\right|^{2}} \psi(y) d \sigma(y) \\
= & -\frac{i \omega}{4} \int_{\partial \Omega} H_{0}^{(1)^{\prime}}\left(\omega\left|y-z_{0}\right|\right)\left|y-z_{0}\right| \frac{\left\langle y-z_{0}, \nu_{y}\right\rangle-\epsilon\left\langle x-\delta \nu_{z_{0}}, \nu_{y}\right\rangle}{\left|y-z_{0}-\epsilon\left(x-\delta \nu_{z_{0}}\right)\right|^{2}} \psi(y) d \sigma(y)+O(\epsilon), \\
:= & I(\epsilon, x)+O(\epsilon) \quad \text { as } \epsilon \rightarrow 0 .
\end{aligned}
$$


Since

$$
\left|y-z_{0}-\epsilon\left(x-\delta \nu_{z_{0}}\right)\right|^{2}=\left|y-z_{0}\right|^{2}+\epsilon^{2}\left|x-\delta \nu_{z_{0}}\right|^{2}-2 \epsilon\left\langle y-z_{0}, x-\delta \nu_{z_{0}}\right\rangle,
$$

it follows that

$$
\begin{aligned}
I(\epsilon, x)-\mathcal{K}_{\Omega}^{\omega} \psi\left(z_{0}\right)= & \int_{\partial \Omega} \frac{\epsilon}{\left|y-z_{0}-\epsilon\left(x-\delta \nu_{z_{0}}\right)\right|^{2}} \Psi_{1}(y) d \sigma(y) \\
& +\epsilon\left|x-\delta \nu_{z_{0}}\right|^{2} \int_{\partial \Omega} \frac{\epsilon}{\left|y-z_{0}-\epsilon\left(x-\delta \nu_{z_{0}}\right)\right|^{2}} \Psi_{2}(y) d \sigma(y),
\end{aligned}
$$

where

$$
\begin{aligned}
\Psi_{1}(y)= & \frac{-i \omega}{4} H_{0}^{(1)^{\prime}}\left(\omega\left|y-z_{0}\right|\right)\left|y-z_{0}\right|\left[2\left\langle y-z_{0}, x-\delta \nu_{z_{0}}\right\rangle \frac{\left\langle y-z_{0}, \nu_{y}\right\rangle}{\left|y-z_{0}\right|^{2}}\right. \\
& \left.\left.-\left\langle x-\delta \nu_{z_{0}}, \nu_{y}\right)\right\rangle\right] \psi(y)
\end{aligned}
$$

and

$$
\Psi_{2}(y)=\frac{i \omega}{4} H_{0}^{(1)^{\prime}}\left(\omega\left|y-z_{0}\right|\right)\left|y-z_{0}\right| \frac{\left\langle y-z_{0}, \nu_{y}\right\rangle}{\left|y-z_{0}\right|^{2}} \psi(y) .
$$

By using the fact that $-\frac{i \omega}{4} H_{0}^{(1)^{\prime}}\left(\omega\left|y-z_{0}\right|\right)\left|y-z_{0}\right| \rightarrow \frac{1}{2 \pi}$ as $\left|y-z_{0}\right| \rightarrow 0$, we deduce the following identities:

$$
\Psi_{1}\left(z_{0}\right)=-\frac{1}{2 \pi}\left\langle x-\delta \nu_{z_{0}}, \nu_{z_{0}}\right\rangle \psi\left(z_{0}\right), \quad \Psi_{2}\left(z_{0}\right)=-\frac{1}{4 \pi} \tau\left(z_{0}\right) \psi\left(z_{0}\right),
$$

where $\tau\left(z_{0}\right)$ is the curvature at $z_{0} \in \partial \Omega$. Applying Lemma 3.10 we conclude that

$$
I(\epsilon, x)=\left(\frac{1}{2} I+\mathcal{K}_{\Omega}^{\omega}\right) \psi\left(z_{0}\right)+o(1) \quad \text { as } \epsilon \rightarrow 0,
$$

which completes the proof of the lemma.

Let $x, y \in \partial B$. Writing $z_{0}+\epsilon\left(x-\delta \nu_{z_{0}}\right)-\left(z_{0}+\epsilon\left(y-\delta \nu_{z_{0}}\right)\right)=\epsilon(x-y)$, the following asymptotic formula holds:

$$
\begin{aligned}
\mathcal{S}_{D}^{\omega}(\varphi)\left(z_{0}+\epsilon\left(x-\delta \nu_{z_{0}}\right)\right)= & \frac{1}{2 \pi} \ln (\gamma \epsilon \omega) \int_{\partial B} \tilde{\varphi}(y) d \sigma(y)+\frac{1}{2 \pi} \int_{\partial B} \ln |x-y| \tilde{\varphi}(y) d \sigma(y) \\
& +O\left((\epsilon \omega)^{2} \ln (\epsilon \omega)\right),
\end{aligned}
$$

where $\tilde{\varphi}(x)=\epsilon \varphi(\epsilon x+z), x \in \partial B$.

Let $x \in \partial \Omega$. We have

$$
\begin{aligned}
\mathcal{S}_{D}^{\omega}(\varphi)(x) & =-\frac{i}{4} \int_{\partial D} H_{0}^{(1)}(\omega|x-y|) \varphi(y) d \sigma(y) \\
& =-\frac{i}{4} \int_{\partial B} H_{0}^{(1)}\left(\omega\left|x-z_{0}-\epsilon\left(y-\delta \nu_{z_{0}}\right)\right|\right) \tilde{\varphi}(y) d \sigma(y) .
\end{aligned}
$$

We conclude, after a lengthy but simple calculation, that in the case of a perfectly conducting inclusion nearly touching the boundary, the leading-order term in the asymptotic expansions of characteristic values of the operator-valued function

$$
\omega \mapsto\left(\begin{array}{cc}
\left(\frac{1}{2} I-\mathcal{K}_{\Omega}^{\omega}\right) & -\mathcal{S}_{D}^{\omega} \\
\mathcal{D}_{\Omega}^{\omega} & \mathcal{S}_{D}^{\omega}
\end{array}\right)
$$

is exactly the one given in the previous section. Thus, the following asymptotic expansion holds. 
Theorem 3.12. Suppose that $\partial B$ and $\partial \Omega$ are of class $\mathcal{C}^{2}$ and $D=\epsilon B+z$, where $z \in \Omega$ is such that $\operatorname{dist}(z, \partial \Omega)=\delta \epsilon$. Let $z_{0}$ be the normal projection of $z$ onto $\partial \Omega$. Then

$$
\mu_{j}^{\epsilon}-\mu_{j}=-\frac{2 \pi}{\ln \left(\frac{\epsilon \sqrt{\mu_{j}}}{\operatorname{cap}(\partial B)}\right)}\left|u_{j}\left(z_{0}\right)\right|^{2}+o\left(\frac{1}{\ln (\epsilon)}\right) .
$$

\section{Asymptotic formula of the eigenvalues in the PRESence OF A CONDUCTIVITY INCLUSION}

In this section we provide a rigorous derivation of a full asymptotic formula for perturbations in the eigenvalues caused by the presence of a conductivity inclusion of small diameter with conductivity different from the one of the background.

Suppose that $D \Subset \Omega$ is of conductivity equal to some positive constant $k \neq 1$. Let $0<\mu_{1}^{\epsilon} \leq \mu_{2}^{\epsilon} \leq \ldots$ be the eigenvalues of $-\nabla \cdot(1+(k-1) \chi(D)) \nabla$ in $\Omega$, where $\chi(D)$ denotes the indicator function of $D$, with the Neumann condition on $\partial \Omega$. We arrange them repeatedly according to their multiplicity.

Fix $j$ and suppose that the unperturbed eigenvalue $\mu_{j}$ is simple. Then there exists a simple eigenvalue $\mu_{j}^{\epsilon}$, near $\mu_{j}$, associated to the normalized eigenfunction $u_{j}^{\epsilon}$, that is, $u_{j}^{\epsilon}$ satisfies the following problem:

$$
\begin{cases}\Delta u+\omega^{2} u=0 & \text { in } \Omega \backslash \bar{D}, \\ \Delta u+\frac{\omega^{2}}{k} u=0 & \text { in } D, \\ \left.u\right|_{+}-\left.u\right|_{-}=0 & \text { on } \partial D, \\ \left.\frac{\partial u}{\partial \nu}\right|_{+}-\left.k \frac{\partial u}{\partial \nu}\right|_{-}=0 & \text { on } \partial D, \\ \frac{\partial u}{\partial \nu}=0 & \text { on } \partial \Omega,\end{cases}
$$

with $\omega=\sqrt{\mu_{j}^{\epsilon}}$.

Once again from [5, we know that the solution of (4.1) can be represented as

$$
u(x)= \begin{cases}\mathcal{D}_{\Omega_{\omega}^{(}}^{\omega}\left(\left.u\right|_{\partial \Omega}\right)(x)+\mathcal{S}_{\Omega}^{\omega}(\phi)(x) & \text { in } \Omega \backslash \bar{D}, \\ \mathcal{S}_{D}^{\sqrt{k}}(\theta)(x) & \text { in } D\end{cases}
$$

where the triplet of densities $\left(\psi:=\left.u\right|_{\partial \Omega}, \phi, \theta\right) \in L^{2}(\partial \Omega) \times L^{2}(\partial D) \times L^{2}(\partial D)$ satisfies the following system of integral equations:

$$
\begin{cases}\left(\frac{1}{2} I-\mathcal{K}_{\Omega}^{\omega}\right)(\psi)(x)-\mathcal{S}_{D}^{\omega}(\phi)(x)=0, & x \in \partial \Omega, \\ \mathcal{D}_{\Omega}^{\omega}(\psi)(x)+\mathcal{S}_{D}^{\omega}(\phi)(x)-\mathcal{S}_{D}^{\frac{\omega}{\sqrt{k}}}(\theta)(x)=0, & x \in \partial D, \\ \epsilon\left[\left.\frac{\partial}{\partial \nu}\left(\mathcal{D}_{\Omega}^{\omega}(\psi)(x)+\mathcal{S}_{D}^{\omega}(\phi)(x)\right)\right|_{+}-\left.k \frac{\partial}{\partial \nu}\left(\mathcal{S}_{D}^{\frac{\omega}{\sqrt{k}}}(\theta)(x)\right)\right|_{-}\right]=0, & x \in \partial D,\end{cases}
$$

for $\omega=\sqrt{\mu_{j}^{\epsilon}}$.

As before, by using the jump formula (2.2), we reduce the problem to the calculation of the asymptotic expressions of the characteristic values of the operator-valued function $\mathcal{A}_{\epsilon}^{d}(\omega)$ given by

$$
\omega \mapsto \mathcal{A}_{\epsilon}^{d}(\omega):=\left(\begin{array}{ccc}
\frac{1}{2} I-\mathcal{K}_{\Omega}^{\omega} & -\mathcal{S}_{D}^{\omega} & 0 \\
\mathcal{D}_{\Omega}^{\omega} & \mathcal{S}_{D}^{\omega} & -\mathcal{S}_{D}^{\frac{\omega}{\sqrt{k}}} \\
\epsilon \frac{\partial}{\partial \nu} \mathcal{D}_{\Omega}^{\omega} & \epsilon\left(\frac{1}{2} I+\left(\mathcal{K}_{D}^{\omega}\right)^{*}\right) & -\epsilon k\left(-\frac{1}{2} I+\left(\mathcal{K}_{D}^{\frac{\omega}{\sqrt{k}}}\right)^{*}\right)
\end{array}\right) .
$$


We shall expand the operator-valued function $\mathcal{A}_{\epsilon}^{d}(\omega)$ in terms of $\epsilon$. With Lemma 3.1 on hand, we only need to write the expansion of $\partial \mathcal{D}_{\Omega}^{\omega} / \partial \nu$ and $\left(\mathcal{K}_{D}^{\omega}\right)^{*}$. On the one hand, we have, for $\psi \in L^{2}(\partial \Omega)$,

$$
\frac{\partial}{\partial \nu} \mathcal{D}_{\Omega}^{\omega}(\psi)(\epsilon x+z)=\sum_{n=1}^{+\infty} \epsilon^{n} \sum_{|\alpha|=n} \frac{1}{\alpha !} \partial^{\alpha} \mathcal{D}_{\Omega}^{\omega}(\psi)(z) \frac{\partial x^{\alpha}}{\partial \nu}, \quad x \in \partial B, \quad d=2,3 .
$$

On the other hand, using the Taylor expansion, we get

$$
\frac{\partial}{\partial \nu_{x}} \Gamma_{\omega}(\epsilon(x-y))=\left\{\begin{array}{c}
\frac{\left\langle x-y, \nu_{x}\right\rangle}{2 \pi \epsilon|x-y|^{2}}\left[1+\sum_{n=1}^{+\infty}(-1)^{n} \frac{(\omega \epsilon)^{2 n}}{2^{2 n} n !(n-1) !}|x-y|^{2 n}\right. \\
\left.\quad \times\left(\ln (\omega \epsilon|x-y|)+\ln \gamma+\frac{1}{2 n}-\sum_{j=1}^{n} \frac{1}{j}\right)\right], d=2, \\
-\frac{\left\langle x-y, \nu_{x}\right\rangle}{4 \pi \epsilon|x-y|^{2}}\left[-\frac{1}{\epsilon|x-y|}\right. \\
\left.+\sum_{n=0}^{+\infty}\left(\frac{1}{n !}-\frac{1}{(n+1) !}\right)(i \omega)^{n+1} \epsilon^{n}|x-y|^{n}\right], d=3,
\end{array}\right.
$$

and we obtain the following expansion.

Lemma 4.1. Let $\varphi \in L^{2}(\partial D)$. Define $\tilde{\varphi}(x)=\epsilon \varphi(\epsilon x+z), x \in \partial B$. Then, for $x \in \partial B$, we have

$$
\begin{aligned}
& \epsilon\left(\mathcal{K}_{D}^{\omega}\right)^{*}(\varphi)(\epsilon x+z)=\mathcal{K}_{B}^{*}(\tilde{\varphi})(x)+\sum_{n=1}^{+\infty}(-1)^{n} \frac{(\omega \epsilon)^{2 n}}{2^{2 n+1} \pi n !(n-1) !} \\
& \quad \times \int_{\partial B}\left\langle x-y, \nu_{x}\right\rangle|x-y|^{2(n-1)}\left(\ln (\omega \epsilon|x-y|)+\ln \gamma+\frac{1}{2 n}-\sum_{j=1}^{n} \frac{1}{j}\right) \tilde{\varphi}(y) d \sigma(y),
\end{aligned}
$$

for $d=2$, while for $d=3$,

$$
\begin{aligned}
& \epsilon\left(\mathcal{K}_{D}^{\omega}\right)^{*}(\varphi)(\epsilon x+z)=\mathcal{K}_{B}^{*}(\tilde{\varphi})(x) \\
& \quad-\frac{1}{4 \pi} \sum_{n=1}^{+\infty}\left(\frac{1}{n !}-\frac{1}{(n+1) !}\right)(i \omega \epsilon)^{n+1} \int_{\partial B}\left\langle x-y, \nu_{x}\right\rangle|x-y|^{n-2} \tilde{\varphi}(y) d \sigma(y),
\end{aligned}
$$

where

$$
\mathcal{K}_{B}^{*}(\tilde{\varphi})(x):=\frac{1}{2(d-1) \pi} \text { p.v. } \int_{\partial B} \frac{\left\langle x-y, \nu_{x}\right\rangle}{|x-y|^{d}} \tilde{\varphi}(y) d \sigma(y) .
$$

As before, define $\tilde{\phi}(x)=\epsilon \phi(\epsilon x+z)$ and $\tilde{\theta}(x)=\epsilon \theta(\epsilon x+z), x \in \partial B$. By Lemma 3.1 and Lemma 4.1, (4.2) now takes the form

$$
\mathcal{A}_{\epsilon}^{d}(\omega)\left(\begin{array}{c}
\psi \\
\tilde{\phi} \\
\tilde{\theta}
\end{array}\right)=0
$$

where

$$
\mathcal{A}_{\epsilon}^{3}(\omega)=\sum_{n=0}^{+\infty}(\omega \epsilon)^{n} \mathcal{A}_{n}^{3}(\omega)
$$


with

$$
\mathcal{A}_{0}^{3}(\omega):=\left(\begin{array}{ccc}
\frac{1}{2} I-\mathcal{K}_{\Omega}^{\omega} & 0 & 0 \\
\mathcal{D}_{\Omega}^{\omega}(\cdot)(z) & \mathcal{S}_{B}^{(0)} & -\mathcal{S}_{B}^{(0)} \\
0 & \frac{1}{2} I+\mathcal{K}_{B}^{*} & -k\left(-\frac{1}{2} I+\mathcal{K}_{B}^{*}\right)
\end{array}\right),
$$

and, for $n \geq 1$, writing $\mathcal{A}_{n}^{3}(\omega)=\left(\left(\mathcal{A}_{n}^{3}(\omega)\right)_{l l^{\prime}}\right)_{l, l^{\prime}=1,2,3}$, we have

$$
\begin{gathered}
\left(\mathcal{A}_{n}^{3}(\omega)\right)_{11}=\left(\mathcal{A}_{n}^{3}(\omega)\right)_{13}=0 \\
\left(\mathcal{A}_{n}^{3}(\omega)\right)_{12}=(-1)^{n} \omega^{-n} \sum_{|\alpha|=n-1} \frac{1}{\alpha !} \partial^{\alpha} \Gamma_{\omega}(x-z)\left(\int_{\partial B} y^{\alpha} \cdot d \sigma(y)\right) \\
\left(\mathcal{A}_{n}^{3}(\omega)\right)_{22}=-\frac{1}{4 \pi} \frac{1}{n !} i^{n} \int_{\partial B}|x-y|^{n-1} \cdot d \sigma(y) \\
\left(\mathcal{A}_{n}^{3}(\omega)\right)_{23}=\frac{1}{4 \pi} \frac{1}{n !}\left(\frac{i}{\sqrt{k}}\right)^{n} \int_{\partial B}|x-y|^{n-1} \cdot d \sigma(y) \\
\left(\mathcal{A}_{n}^{3}(\omega)\right)_{21}=\frac{1}{\omega^{n}} \sum_{|\alpha|=n} \frac{1}{\alpha !} \partial^{\alpha} \mathcal{D}_{\Omega}^{\omega}(\cdot)(z) x^{\alpha} \\
\left(\mathcal{A}_{n}^{3}(\omega)\right)_{31}=\frac{1}{\omega^{n}} \sum_{|\alpha|=n-1} \frac{1}{\alpha !} \partial^{\alpha} \mathcal{D}_{\Omega}^{\omega}(\cdot)(z) \frac{\partial x^{\alpha}}{\partial \nu}
\end{gathered}
$$

$\left(\mathcal{A}_{1}^{3}(\omega)\right)_{32}=\left(\mathcal{A}_{1}^{3}(\omega)\right)_{33}=0$, and

$$
\begin{aligned}
& \left(\mathcal{A}_{n}^{3}(\omega)\right)_{32}=-\frac{1}{4 \pi}\left(\frac{1}{(n-1) !}-\frac{1}{n !}\right)(i)^{n} \int_{\partial B}\left\langle x-y, \nu_{x}\right\rangle|x-y|^{n-3} \cdot d \sigma(y), \\
& \left(\mathcal{A}_{n}^{3}(\omega)\right)_{33}=\frac{k}{4 \pi}\left(\frac{1}{(n-1) !}-\frac{1}{n !}\right)\left(\frac{i}{\sqrt{k}}\right)^{n} \int_{\partial B}\left\langle x-y, \nu_{x}\right\rangle|x-y|^{n-3} \cdot d \sigma(y),
\end{aligned}
$$

for $n \geq 2$. Similarly, one can compute $\mathcal{A}_{\epsilon}^{2}(\omega)$.

It has been shown in [5] that

$$
\left(\begin{array}{cc}
\mathcal{S}_{B}^{(0)} & -\mathcal{S}_{B}^{(0)} \\
\frac{1}{2} I+\mathcal{K}_{B}^{*} & -k\left(-\frac{1}{2} I+\mathcal{K}_{B}^{*}\right)
\end{array}\right)
$$

is invertible. In fact, the inverse is given by

$$
\frac{1}{k-1}\left(\begin{array}{ll}
k\left(\lambda I-\mathcal{K}_{B}^{*}\right)^{-1}\left(\frac{1}{2} I-\mathcal{K}_{B}^{*}\right)\left(\mathcal{S}_{B}^{(0)}\right)^{-1} & \left(\lambda I-\mathcal{K}_{B}^{*}\right)^{-1} \\
-\left(\lambda I-\mathcal{K}_{B}^{*}\right)^{-1}\left(\frac{1}{2} I+\mathcal{K}_{B}^{*}\right)\left(\mathcal{S}_{B}^{(0)}\right)^{-1} & \left(\lambda I-\mathcal{K}_{B}^{*}\right)^{-1}
\end{array}\right),
$$

where $\lambda:=\frac{k+1}{2(k-1)}$. Therefore the invertibility of $\mathcal{A}_{0}^{3}(\omega)$ holds for any $\omega \notin\left\{\sqrt{\mu_{j}}\right\}_{j \geq 1}$.

The asymptotic expansion can now be constructed in exactly the same manner as in Theorem 3.6 and the formula is exactly the same as (3.6). Analogously, the two-dimensional case can be treated without any new difficulty.

In the same way as in Section 3, we can recover the following result from [12, [11] giving the leading-order term in the asymptotic expansion of the eigenvalue perturbations.

Corollary 4.2. Suppose $\mu_{j}$ is a simple eigenvalue associated with the orthonormal eigenfunction $u_{j}$. The following asymptotic expansion holds:

$$
\mu_{j}^{\epsilon}-\mu_{j}=\epsilon^{d} \nabla u_{j}(z) \cdot M(k, B) \nabla u_{j}(z)+o\left(\epsilon^{d}\right),
$$


where

$$
M(k, B):=\int_{\partial B}\left(\frac{k+1}{2(k-1)} I-\mathcal{K}_{B}^{*}\right)^{-1}(\nu) y d \sigma(y)
$$

is the so-called polarization tensor associated with the domain $B$ and the conductivity k. See [4, 6].

\section{CONCluding REMARKS}

We conclude this paper by making a few remarks. If we consider the eigenvalue problem in the presence of a diametrically small conductivity inclusion that is nearly touching the boundary, then following the arguments given in Subsection 3.2, we can easily show that the leading-order term in the asymptotic expansion of the eigenvalue perturbations is the same as in Corollary 4.2. We leave the details to the reader.

The results and the methods of this paper have been extended to derive complete asymptotic expansions of eigenvalue perturbations for the Lamé system in [8]. They have also been used in [10, 9] to investigate the band-gap structures of the frequency spectrum for both electromagnetic and elastic waves in high-contrast, two-component periodic media and have given a new tool for the optimal design problem in photonic and phononic crystals.

Based on a small volume expansion of the variations of the modal parameters that are due to the presence of a small inclusion, we have designed in [7] an efficient anomaly detection algorithm from modal measurements which consists of minimizing a functional whose minimizer yields the location and the size of the anomaly. The numerical results performed in [7 clearly demonstrate that our detection algorithm works well even when the material parameter of the inclusion is not a priori known.

\section{ACKNOWLEDGEMENTS}

This research was partly supported by CNRS-KOSEF grant No. 14889, Korea Science and Engineering Foundation grant R01-2006-000-10002-0, ACI Nouvelles Interfaces des Mathématiques No. 171, and the ANR project EchoScan. The authors would also like to thank Hyundae Lee for carefully reading an early version of this paper.

\section{REFERENCES}

[1] J. Albert, Generic properties of eigenfunctions of elliptic partial differential operators, Trans. Amer. Math. Soc., 238 (1978), 341-354. MR0471000 (57:10743)

[2] H. Ammari, M. Asch, and H. Kang, Boundary voltage perturbations caused by small conductivity inhomogeneities nearly touching the boundary, Adv. Appl. Math., 35 (2005), 368-391. MR2174737 (2006g:35033)

[3] H. Ammari, N. Béreux, and J.C. Nédélec, Resonances for Maxwell's equations in a periodic structure, Japan J. Indust. Appl. Math., 17 (2000), 149-198. MR1769190 (2001d:78012)

[4] H. Ammari and H. Kang, Reconstruction of Small Inhomogeneities from Boundary Measurements, Lecture Notes in Mathematics, Vol. 1846, Springer-Verlag, Berlin, 2004. MR 2168949 (2006k:35295)

[5] H. Ammari and H. Kang, Boundary layer techniques for solving the Helmholtz equation in the presence of small inhomogeneities, J. Math. Anal. Appl., 296 (2004), 190-208. MR 2070502 (2005c:35054)

[6] H. Ammari and H. Kang, Polarization and Moment Tensors with Applications to Inverse Problems and Effective Medium Theory, Applied Mathematical Sciences, Vol. 162, SpringerVerlag, New York, 2007. MR2327884 
[7] H. Ammari, H. Kang, E. Kim, and H. Lee, Vibration testing for anomaly detection, Math. Meth. Appl. Sci., to appear.

[8] H. Ammari, H. Kang, and H. Lee, Asymptotic expansions for eigenvalues of the Lamé system in the presence of small inclusions, Comm. Part. Diff. Equat., 32 (2007), 1715-1736. MR2372485 (2008k:35464)

[9] H. Ammari, H. Kang, and H. Lee, Asymptotic analysis of high-contrast phononic crystals and a criterion for the band-gap opening, preprint.

[10] H. Ammari, H. Kang, S. Soussi, and H. Zribi, Layer potential techniques in spectral analysis. Part II: Sensitivity analysis of spectral properties of high contrast band-gap materials, Mult. Model. Simul., 5 (2006), 646-663. MR2247766 (2007k:81234)

[11] H. Ammari and A. Khelifi, Electromagnetic scattering by small dielectric inhomogeneities, J. Math. Pures Appl., 82 (2003), 749-842. MR2005296 (2005a:35262)

[12] H. Ammari and S. Moskow, Asymptotic expansions for eigenvalues in the presence of small inhomogeneities, Math. Meth. Appl. Sci. 26 (2003), 67-75. MR1943110 (2003j:35035)

[13] H. Ammari and F. Triki, Resonances for microstrip transmission lines, SIAM J. Appl. Math., 64 (2004), 601-636. MR2049666 (2005c:35053)

[14] H. Ammari and F. Triki, Splitting of resonant and scattering frequencies under shape deformation, J. Diff. Equat., 202 (2004), 231-255. MR2068440(2006a:35220)

[15] G. Besson, Comportement asymptotique des valeurs propres du laplacien dans un domaine avec un trou, Bull. Soc. Math France 113 (1985), 211-230. MR820320 (87d:58143)

[16] G. Courtois, Spectrum of manifolds with holes, J. Funct. Anal. 134 (1995), 194-221. MR1359926 (97b:58142)

[17] D. Daners, Dirichlet problems on varying domains, J. Diff. Equat. 188 (2003), 591-624. MR1955096 (2004a:35042)

[18] R.R. Gadyl'shin and A.M. Il'in, Asymptotic behavior of the eigenvalues of the Dirichlet problem in a domain with a narrow slit, Sbornik Math. 189 (1998), 503-526. MR1632410 (99k:35138)

[19] I. C. Gohberg and E. I. Sigal, An operator generalization of the logarithmic residue theorem and Rouché's theorem, Mat. Sb. (N.S.) 84 (1971), 607-642. MR.0313856 (47:2409)

[20] T. Kato, Perturbation Theory for Linear Operators, Springer-Verlag, New York, 1976. MR0407617 (53:11389)

[21] V.G. Maz'ya, S.A. Nazarov, and B.A. Plamenevskiı̌, Asymptotic expansions of eigenvalues of boundary value problems for the Laplace operator in domains with small openings, Math. USSR 24 (1985), 321-346. MR0740795 (86b:35152)

[22] I. McGillivray, Capacitary asymptotic expansion of the groundstate to second order, Comm. Partial Diff. Equat. 23 (1998), 2219-2252. MR.1662184 (2000j:47047)

[23] A. Noll, Domain perturbations, shift of eigenvalues and capacity, J. Funct. Anal. 170 (2000), 246-263. MR1736203 (2000m:47061)

[24] S. Osher and F. Santosa, Level set methods for optimization problems involving geometry and constraints. I. Frequencies of a two-density inhomogeneous drum, J. Comput. Phys., 171 (2001), 272-288. MR.1843648 (2002f:65088)

[25] S. Ozawa, Singular variation of domains and eigenvalues of the Laplacian, Duke Math. J. 48 (1981), 767-778. MR782576 (86k:35117)

[26] S. Ozawa, Electrostatic capacity and eigenvalues of the Laplacian, J. Fac. Sci. Univ. Tokyo, Sect IA 30 (1983), 53-62. MR700595 (84m:35093)

[27] S. Ozawa, An asymptotic formula for the eigenvalues of the Laplacian in a three-dimensional domain with a small hole, J. Fac. Sci. Univ. Tokyo, Sect IA 30 (1983), 243-257. MR.722496 (85k:35173)

[28] S. Ozawa, Spectra of domains with small spherical Neumann boundary, J. Fac. Sci. Univ. Tokyo, Sect IA 30 (1983), 259-277. MR722497 (85k:35174)

[29] S. Ozawa, Asymptotic property of an eigenfunction of the Laplacian under singular variation of domains-the Neumann condition, Osaka J. Math. 22 ( 1985), 639-655. MR815438 (87i:35165)

[30] S. Ozawa, Eigenvalues of the Laplacian under singular variation of domains-the Robin problem with obstacle of general shape, Proc. Japan Acad. Ser. A Math. Sci. 72 (1996), 124-125. MR.1404488 (97e:35037)

[31] J. Rauch and M. Taylor, Potential and scattering theory on wildly perturbed domains, J. Funct. Anal. 18 (1975), 27-59. MR0377303 (51:13476) 
[32] G.F. Roach, Green's Functions: Introductory Theory with Applications, Van Nostrand Reinhold, London, 1970. MR0277804 (43:3537)

[33] J. Sanchez Hubert and E. Sanchez Palencia, Vibration and Coupling of Continuous Systems, Springer-Verlag, 1989. MR996423 (91c:00018)

[34] M.E. Taylor, Partial Differential Equations. II. Qualitative Studies of Linear Equations, Applied Mathematical Sciences 116, Springer-Verlag, 1996. MR1395149 (98b:35003)

[35] K. Uhlenbeck, Generic properties of eigenfunctions, Amer. J. Math., 98 (1976), 1059-1078. MR0464332 (57:4264)

[36] G.C. Verchota, Layer potentials and boundary value problems for Laplace's equation in Lipschitz domains, J. Funct. Anal., 59 (1984), 572-611. MR769382 (86e:35038)

[37] M.J. Ward and J.B. Keller, Strong localized perturbations of eigenvalue problems, SIAM J. Appl. Math. 53 (1993), 770-798. MR.1218383 (94h:35044)

Centre de Mathématiques Appliquées, CNRS UMR 7641, École Polytechnique, 91128 Palaiseau Cedex, France

E-mail address: ammari@cmapx.polytechnique.fr

Department of Mathematical Sciences and Rim, Seoul National University, Seoul 151-747, KOREA

E-mail address: hkang@math.snu.ac.kr

Current address: Department of Mathematics, Inha University, Incheon, 402-751, Korea

E-mail address: hbkang@inha.ac.kr

Department of Mathematics, Colorado State University, Fort Collins, Colorado 80523

E-mail address: lim@math.colostate.edu

Current address: Department of Mathematics, Korean Advanced Institute of Science and Technology, Daejeon 305-701, Korea

E-mail address: mklim@kaist.ac.kr

Centre de Mathématiques Appliquées, CNRS UMR 7641, École Polytechnique, 91128 Palaiseau Cedex, France

E-mail address: zribi@cmapx.polytechnique.fr

Current address: Department of Mathematics, Korean Advanced Institute of Science and Technology, Daejeon 305-701, Korea 